\title{
ENFOQUE CURRICULAR CENTRADO EN LA PERSONA
}

\author{
Luis Manuel Jiménez Castro \\ Estudiante de la Maestría en Planificación Curricular \\ Universidad de Costa Rica \\ San José, Costa Rica
}

Recibido: 27-XI-2007 • Aceptado 9-IV-2008 • Corregido 23-V-2008

\begin{abstract}
Resumen: Un enfoque curricular estructura, visualiza y organiza el proceso educativo. Sin embargo, a pesar de su importancia, el sistema educativo costarricense no identifica unas características prácticas claras de un enfoque curricular, por medio del cual el educador pueda ubicar el tipo de estudiante que debe formar.

Se desea sistematizar y aportar por medio de una revisión bibliográfica, del enfoque curricular centrado en la persona, una alternativa que favorezca la formación de las personas de manera integral, que promueva la crítica, la búsqueda de soluciones a problemas comunales y en general al avance de la sociedad a través del bien común.
\end{abstract}

Palabras clave: Enfoque curricular, educación personalizada, currículo.

\section{Introducción}

El currículo es un proceso social, por lo que en su elaboración intervienen una gran cantidad de elementos que se deben tomar en cuenta. Algunos de estos son el individuo, la sociedad, las decisiones políticas, el contexto entre otros. Los distintos elementos que se encuentran en el desarrollo del currículo, presentarán múltiples relaciones entre sí, la interpretación que se haga de ellos; la importancia que se le asigne a cada elemento, los elementos que se tomen como ejes centrales del mismo; son decisiones relacionadas directamente con distintas formas de ver la realidad, siempre bajo la luz de disciplinas como la filosofía, psicología, sociología y todas aquellas ciencias sociales que permitan de una $\mathrm{u}$ otra forma clarificar dicha realidad para su análisis.

Así, al conjunto formado por los distintos puntos de vista de la realidad, las relaciones entre los elementos del currículo, la organización que se realice del currículo con base en ellos y los distintos fundamentos teóricos, se conoce como enfoque curricular.

La importancia de los enfoques curriculares radica en que cada uno de ellos va 


\begin{abstract}
A curricular approach structures, visualizes, and organizes the educational process. However in spite of their importance, the Costa Rican educational system does not identify some clear characteristics of a curricular approach, by means of which the educator can locate the student type they should form.

This article pretends to systematize and to contribute by means of a bibliographical review of a curricular approach centered in the person, an alternative that allows the formation of people in an integral way, promoting criticism, searching solutions to communal problems, and in general, the advance of the society through the common wellbeing.
\end{abstract}

Key words: curricular approach, personalized approach, curriculum. a orientar el currículo en todas sus partes, por ejemplo Castillo (2003) indica que un enfoque curricular es la "forma como se estructura, visualiza, organiza y se ejecuta el proceso educativo" por su parte Molina (2004, p. 24) indica que es una "posición teórica que se adopta y desde la cual se caracterizan los elementos y los procesos curriculares".

Se puede apreciar cómo el enfoque curricular resulta necesario, si se desea dar coherencia al proceso en general, pues todo el tramado social que se desarrolla en el proceso educativo debe tener alguna estructura, la cual se determina por la forma en que se visualizan sus componentes.

Un ejemplo de la importancia que tiene el enfoque curricular en la coherencia de todo diseño de esta naturaleza, es presentado por Zabalza (1997) al referirse a los contenidos de la enseñanza, este autor plantea que proponer dichos contenidos no es únicamente responder al qué enseñar, sino que detrás de su selección, existe todo un posicionamiento en la toma de decisiones, condicionado por una serie de posturas previas respecto a qué y para qué es la escuela, qué peso ha de adquirir cada disciplina, con qué actitud habrá que acercarse a los conocimientos, aspectos todos, que se definen de acuerdo con el enfoque curricular que se haya elegido previamente.

Los enfoques curriculares suelen ser llamados también concepciones curriculares, éstas suelen orientarse hacia dos grandes áreas, una que da énfasis al objeto y otra que va a dar importancia a la persona. La primera se caracteriza por una atención prioritaria del currículo en lo que se refiere a las tareas del conocimiento, los métodos, las técnicas, los sucesos educativos, los procedimientos. Esta concepción curricular ha predominado históricamente en los programas educativos; pues responde muy bien a la eficiencia que se exige en la actualidad a cada una de las acciones que se realizan en las instituciones educativas, como parte de organizaciones donde el logro de metas concretas en poco tiempo resulta 
muy importante, los enfoques que se orienten de esta manera van a enfatizar en la transformación del medio, la transmisión del conocimiento, en los medios educativos más prácticos y eficientes en la consecución de objetivos palpables, en la enseñanza, en el rendimiento reflejado por estadísticas, en la eficiencia, en la productividad; su criterio de evaluación será de carácter cuantitativo.

Por otra parte el área curricular que da importancia a la persona se caracteriza por establecer una clara intencionalidad del tipo de hombre y mujer que se desea formar, va a reflejar la importancia de alcanzar su desarrollo personal de manera integral.

Se preocuparán por lo tanto de las vivencias, la imaginación, la voluntad y todas aquellas cuestiones propias de la persona. Privilegia el aprendizaje, el crecimiento de hombres y mujeres a nivel individual, el desarrollo a escala humana. El propósito de esta tendencia es lograr el rescate del educador y del educando.

Schiro citado por Zabalza (1997) en concordancia con la clasificación anterior identifica dos dimensiones bipolares, una donde prevalecen las consideraciones sobre la realidad objetiva y su contraparte donde lo que predomina es la realidad subjetiva. De estas dos dimensiones se pueden extraer distintas formas de ver la escuela, formas orientadas a las disciplinas, como en un modelo academicista, a la eficacia como en un modelo tecnológico, al estudiante como persona, tal y como se desea en un modelo humanista ó en la búsqueda del cambio social como en los modelos críticos.

Lo anterior realza la importancia, ya mencionada, de tener claro cuál es el enfoque curricular de un proyecto educativo, pues resultará indispensable en la identificación y comprensión de los elementos conceptuales que van a predominar en la posterior toma de decisiones curriculares.

Los enfoques curriculares no siempre se presentan de una manera clara, esto en gran medida por la complejidad que encierran y la forma en que se analizan, por esta razón Palladino (1998) señala algunas preguntas claves que deben responderse para una posterior identificación del enfoque de determinado proyecto curricular, éstas interrogantes se dirigen a ¿qué idea de persona o naturaleza humana se pretende alcanzar?, ¿cuál teoría de aprendizaje es la que se emplea?, ¿qué tipo de relaciones se dan entre los profesores y los estudiantes?, ¿qué importancia se le da al contenido? Y algunas otras en torno a la función que se le asigna a la escuela y su papel en la sociedad.

Hoy en día es necesario que no solo los especialistas en currículo sino todos los que de una u otra manera están relacionados con la educación formal, tengan claro cuáles son los enfoques curriculares que están dando respuesta a preguntas de su labor diaria tales como ¿qué enseñar?, ¿cómo enseñar y ¿cuándo enseñar?

En el presente artículo se ha decidido revisar bibliografía referente al enfoque curricular centrado en la persona como una iniciativa que dé paso a futuros análisis de otros enfoques curriculares. El por qué de ésta revisión bibliográfica, tiene su explicación en el hecho de que los diseños curriculares se orientan con frecuencia más hacia el objeto, siguiendo la tradición curricular del modelo Tyleriano, con objetivos claros que presentan conductas observables, lo que brinda mucho control y donde el papel que se le asigna al docente es el de consumidor curricular, al relegarlo como un simple aplicador de un determinado currículo y no hacerle partícipe en la elaboración del mismo y el papel del estudiante se relega a la simple adquisición de conocimientos, dejando de lado su área afectiva, física y volitiva entre otras áreas que permitan su desarrollo integral.

Este modelo curricular surge en 1949 , Díaz (1997) señala que la idea de Ralph Tyler era la de tecnificar la educación por medio de objetivos conductuales, según él las decisiones en relación con los aprendizajes que deberían promoverse en un 
programa escolar, debían ser producto del análisis de diversas investigaciones realizadas a las fuentes curriculares, es decir a los estudiantes y sus necesidades sobre la sociedad, el análisis de tareas y los procesos culturales y sobre la función y el desarrollo de los contenidos. Para posteriormente poder definir objetivos conductuales relevantes, y que a su vez pudieran ser medidos con claridad, al final del proceso de enseñanza y verificar así su cumplimiento.

\section{Búsqueda de coherencia entre los documentos oficiales y la práctica}

Como se mencionó anteriormente, los diseños curriculares con frecuencia dejan de lado la formación integral de la persona.

Sin embargo, al revisar la misión institucional del Ministerio de Educación Pública se puede encontrar un compromiso de promover el desarrollo y consolidación de un sistema educativo de excelencia, con el fin de permitir el acceso de toda la población a una educación de calidad y destaca que dicha educación será centrada en el desarrollo integral de las personas y en la promoción de una sociedad costarricense integrada por las oportunidades y la equidad social. Si se retoma lo expuesto por Palladino (1998) quien señala que el identificar en documentos oficiales el ideal de ser humano que se desea formar, facilita determinar el enfoque curricular que se desprende del mismo. Se puede concluir que en el sistema educativo costarricense, la concepción de educación que se presenta debería estar orientada hacia la formación de la persona, tal y como lo señala la misión y que se resalta en los fines educativos que buscan lograr lo siguiente:

a) La formación de ciudadanos amantes de su patria, conscientes de sus deberes, de sus derechos y de sus libertades fundamentales, con profundo sentido de responsabilidad y de respeto a la dignidad humana; b) Contribuir al desenvolvimiento de la personalidad humana;

c) Formar ciudadanos para una democracia en que se concilien los intereses del individuo con los de la comunidad;

d) Estimular el desarrollo de la solidaridad y de la comprensión humana; y

e) Conservar y ampliar la herencia cultural, impartiendo conocimientos sobre la historia del hombre, las grandes obras de la literatura y los conceptos filosóficos fundamentales (Ministerio de Educación Pública, s.f., p. 1).

Pero a pesar de lo anterior, el manejo de objetivos conductuales y el uso de pruebas sumativas para comprobar su adquisición, son la constante en el desarrollo de cada curso lectivo, muy al estilo de Tyler, explicado ya en líneas anteriores.

De esta forma se puede indicar que el sistema educativo costarricense no identifica un enfoque curricular bien definido pues aunque en los documentos se propone una dimensión curricular más dirigida hacia la persona, en la práctica del docente y en su máximo punto en las pruebas de bachillerato se aprecia una dimensión más orientada hacia el academicismo, lo cual muestra falta de coherencia con lo que se busca entre el ideal y la práctica.

El educador requiere que precisamente se busque una alternativa clara que permita coherencia entre la práctica y lo prescrito en todo el proceso educativo, que favorezca por lo tanto la formación de las personas de manera integral, pues es poco lo que se logra con una educación preocupada en el manejo de asignaturas cargadas de contenidos, sino se promueve en forma paralela la crítica, la búsqueda de soluciones a problemas comunales y en general al avance de la sociedad a través del bien común.

La educación debe promover los medios necesarios que permitan dar respuesta a intereses individuales y colectivos, a las diferentes formas de aprendizaje e inclusive a los ritmos de aprendizaje de cada estudiante.

Esto es lo que se desea encontrar y sistematizar por medio de una revisión 
bibliográfica, del enfoque curricular centrado en la persona.

\section{Identificación del enfoque curricular y su importancia en el desarrollo de la práctica educativa}

En el presente trabajo se organiza parte de la información que existe sobre el mismo, pues se ha podido apreciar en la revisión de bibliografía, cómo en el tratamiento del tema por parte de algunos autores se puede encontrar el empleo de términos distintos, para referirse a cuestiones similares.

Algunos ejemplos de lo anterior se muestran en la terminología empleada por Chadwick (1987) y Palladino (1998) quienes hablan de un currículo como realización personal, el cual pone énfasis a orientar el proceso educativo en términos de las necesidades de la persona para alcanzar su desarrollo. Por otra parte Villarini (1996) se refiere a un currículo basado en el desarrollo humano, como una concepción humanista de la educación donde lo que importa son las experiencias y necesidades del estudiante. Castillo (2003) utiliza en cambio distintos términos que abarcan a varios autores, él habla de un currículo como experiencia integrada, de realización personal como Chadwick (1987) y Palladino (1998), humanista como la línea de Villarini (1996) y personalizado; García (1993) en cambio utiliza el término educación personalizada para referirse al enfoque curricular centrado en la persona, este autor aborda en su enfoque los valores del humanismo cristiano.

Esta diversidad de términos podría confundir a las personas interesadas en utilizar este enfoque curricular en sus distintos trabajos (planificadores del currículo, estudiantes de la carrera de educación), al pensar que las propuestas de los especialistas en el tema son distintas unas de otras, cuando lo oportuno sería analizar de qué manera se enriquece el enfoque con los aportes que cada autor hace y así eliminar la confusión en el manejo de la información.

Hablar de las características de un enfoque curricular es un tema amplio y complejo, pues en el currículo "se concretan y toman cuerpo una serie de principios pedagógicos, psicopedagógicos e ideológicos, que tomados en su conjunto muestran la orientación general del sistema educativo o de una institución específica" (Coll, 1991, p. 21).

Resulta importante observar dentro de la organización curricular las distintas relaciones entre sus miembros, así como las características de sus roles y sus concepciones sobre lo qué es la educación y sus funciones, para poder así determinar las características del enfoque curricular abordado por la institución. Debe identificarse de esta manera el papel del docente dentro del proceso educativo, el uso que hace de los medios que se pongan a su disposición, la forma en que el diseño del currículo se lleva a la práctica, la importancia que se le dan a los contenidos, las características de la participación del estudiante en el desarrollo del proceso educativo y las características de la evaluación en cuestiones como el qué evaluar, cuál es el objetivo de la evaluación y con qué frecuencia se realizará, todos estos aspectos reflejarán las características propias de un determinado enfoque.

Además de lo anterior es importante analizar cuestiones como la idea de ser humano que se maneja en la institución, si existe una orientación expresa hacia alguna determinada filosofía ó teoría educativa y además establecer la comparación entre los aspectos anteriores y ver si efectivamente se ven reflejados en su proyecto educativo.

De no encontrarse claro un rol del docente y del estudiante en documentos institucionales, se pueden observar dichos roles en la práctica, con observaciones de aula que permitan determinar el papel de 
los actores en el proceso y además analizar aspectos curriculares endógenos en relación a objetivos, contenidos y evaluación. Lo anterior permitirá determinar el enfoque de una institución y sus características, pues los documentos orientadores no siempre explicitan este o proponen un determinado enfoque que no es acorde con la realidad que se vive en el centro.

\section{El enfoque curricular centrado en la persona}

La educación se considera un eslabón importante de la socialización, por otra parte es cada vez más frecuente escuchar comentarios a favor de atender las necesidades individuales, quienes han desarrollado el enfoque curricular centrado en la persona buscan con esta concepción, favorecer el desarrollo humano mediante procesos que integren la individualización y la socialización, aceptando que estos dos aspectos se dan simultáneamente y que a partir de la fusión de los dos, se construye la identidad y el mundo personal. Esta concepción difiere así de la concepción que tradicionalmente se tiene sobre educación individual y educación socializada.

García Hoz (1988a) menciona que la educación colectiva y la educación individual son formas parciales e incompletas de educación. La educación individual aísla a la persona de los compañeros, le impide establecer relaciones sociales de igualdad, de enriquecerse a través de los trabajos en equipo, lo hace dependiente, con lo cual reduce sus posibilidades de desarrollo general.

La educación colectiva se entiende simplemente como la acción del maestro que estimula y dirige la formación de un conjunto de alumnos, un grupo dependiente del educador.

En términos muy generales se puede señalar entonces que esta concepción curricular se encuentra estrechamente enraizada en un amplio cuerpo de "valores universales", por lo que se busca atender a la persona colaborando en la construcción de su proyecto personal de vida, de una manera libre, responsable y auténtica, atendiendo a cada estudiante de manera individual, pero rescatando a su vez la parte social.

Esta descripción general abarca de manera amplia lo propuesto por distintos autores que se han referido al tema. El tocar aspectos tan citados socialmente como el tema de "valores universales", proyecto de vida, libertad y responsabilidad, ha llevado a los autores ha utilizar distintos términos para hablar de dicho enfoque, manteniendo siempre la idea central señalada al inicio de este apartado.

Castillo (2003) refiriéndose a la variedad de términos utilizados para referirse a este enfoque, señala que una posible razón se debe a que el lenguaje empleado, tal y como se ha señalado, es un lenguaje amplio, que debe ser coherente con el lenguaje humanista, con el lenguaje existencialista y con la psicología existencial, los cuales conciben a la educación como un medio que sea de gran motivación al estudiante, con el cual logre responder a sus necesidades individuales de crecimiento y de realización humana plena: realización independiente de los planos cognoscitivo, afectivo y motriz.

A continuación se presentan diferentes términos encontrados en la literatura respecto al enfoque curricular centrado en la persona, con lo que se pretende ir clarificando la variedad de términos a la que Castillo (2003) hace referencia.

Se encuentran así los siguientes términos:

- Currículo como realización personal, término empleado por Chadwick (1987), Palladino (1998) y Castillo (2003).

- Currículo como experiencia integrada, empleado por Castillo (2003).

- Currículo psicologista, utilizado por quienes comparten una línea 
humanista como Vilarini (1996), Molina (2004) y García (2007).

- Currículo como educación personalizada, término empleado por García Hoz (1993).

\section{Currículo como realización personal}

Cuando se hace referencia al enfoque centrado en la persona como realización personal, suele hacerse énfasis a orientar el proceso educativo en términos de las necesidades de la persona para alcanzar su desarrollo.

Palladino (1998) expone que para alcanzar dicha realización personal se puede hacer un énfasis en la adquisición de conocimientos, habilidades y valores humanos o espirituales en general.

Al buscar el desarrollo de la persona son claros los lineamientos de abarcarla en su totalidad, señala la importancia de lograr el desarrollo interno del individuo, aspecto importante pues al buscar la totalidad de la persona no se queda solo en la exposición de lo aprendido por parte de la población estudiantil, es importante dentro de este enfoque curricular incluir en el aprendizaje la realidad de las personas, sus experiencias, la proyección de lo aprendido en su realidad inmediata.

Chadwick (1987) indica que ésta es una posición más complicada de analizar que otras como el racionalismo académico que enfatiza en la transmisión de valores y tradiciones culturales para que los estudiantes dominen las grandes ideas y conceptos que el hombre ha creado o el currículo como proceso tecnológico que se centra únicamente en cómo entregar la información al estudiante.

Castillo (2003) indica que un currículo en esta línea tiene como función fundamental la organización de experiencias personales integradoras y altamente satisfactorias para cada aprendizaje individual.

\section{Currículo como experiencia integrada}

De acuerdo con Castillo (2003) además del término de currículo como experiencia integrada en esta posición se utiliza además los términos de realización personal, humanista ó personalizado, esta variedad de términos son reflejo de ese lenguaje amplio que el mismo Castillo (2003) menciona, al tratar de ser coherente con el humanismo y aspectos de la psicología existencialista.

Dentro de esta línea se ve como objetivo principal la organización de las experiencias del currículo de una manera que lleguen a ser experiencias personales, integrales y que sean de provecho para el aprendizaje individual.

Las actividades que se desarrollan deben favorecer de esta manera un apoyo al fortalecimiento de la personalidad de cada estudiante.

\section{Currículo psicologista}

Señala García (2007) que quien más ha analizado bajo este línea la concepción curricular centrada en la persona es Carl Rogers psicólogo estadounidense quien centró su trabajo en la vivencia global de las situaciones de los grupos a través de las acciones y las emociones.

García (2007) indica que este psicólogo del siglo veinte señala que la población estudiantil desarrollará su aprendizaje cuando éste llegue a ser significativo y esto sucede cuando se involucra a la persona como totalidad, incluyendo sus procesos afectivos y cognitivos, y se desarrolla en forma experimental. Señala que en el desarrollo del currículo en el salón de clases los estudiantes consideren el tema a tratar como algo importante para sus objetivos personales.

Se indica que en esta perspectiva el aprendizaje es mejor si se promueve como 
participativo, donde la población estudiantil decida, mueva sus propios recursos y se responsabilice de lo que va a aprender, el rol del estudiante es activo.

Para lograr este papel activo del estudiante se requiere una persona motivada y llena de confianza para actuar y esas características se alcanzan únicamente en un ambiente de respeto, comprensión y apoyo para los estudiantes, se sugiere por eso que el profesor no utilice recetas estereotipadas, pues la práctica educativa es dinámica, se requiere entonces un papel del docente activo, que se mantenga al lado de los estudiantes, que actúe de manera innovadora, que sea él mismo, que sea auténtico y no un simple consumidor del currículo.

Es una de las posiciones más analizadas, aparece como una posición conciliadora entre dos de los paradigmas predominantes en los Estados Unidos en la década de los cincuenta: el conductismo y el psicoanálisis.

Por otra parte, la incorporación de líneas de orientación humanista en los currículos norteamericanos, aparece como resultado de las protestas por la excesiva deshumanización de éstos y por la falta de consideración a las características particulares de los estudiantes, situaciones que no permitían el desarrollo total de las capacidades de los jóvenes y provocaban fallas en el trabajo académico.

Dentro de esta corriente se busca conciliar además el paradigma humanista y sus efectos directos en el conocimiento y la promoción de los procesos integrales de la persona.

Los humanistas, fuertemente influenciados por las corrientes existencialistas, parten del supuesto de que la personalidad humana es una totalidad en continuo proceso de desarrollo. $\mathrm{Y}$ aunque se considera que para comprender al individuo es importante ubicarlo en su contexto.

Otro de los autores que hace mención al currículo centrado en la persona desde una concepción humanista es Villarini (1996), quien señala que esta concepción humanista convierte en centro de atención la experiencia y necesidades del estudiante a la vez que da mayor peso a su perspectiva biopsicosocial.

En relación con los elementos endógenos del currículo Villarini (1996) menciona que los objetivos y el contenido curricular se orientan a lo que es pertinente y significativo para el proceso de desarrollo del estudiante, lo que conlleva que éstos sean sumamente flexibles y experimentales a la vez que deben estar conectados con la realidad del estudiante y que según él resulta indispensable que durante la elaboración del currículo estén presentes asesores en áreas del desarrollo humano.

Por otra parte Molina (2004) también menciona como elemento central al estudiante, por lo que la práctica pedagógica es una respuesta a las características, necesidades e intereses de los alumnos. Dicha práctica debe estar orientada hacia el respeto de las diferencias individuales y considerar el desarrollo del pensamiento y ritmo de las personas.

Molina (2004) indica que el docente debe recurrir durante sus lecciones a estrategias didácticas activas y participativas. Durante el desarrollo del currículo se debe tener presente siempre, las dimensiones cognitiva, afectiva, social y psicomotriz de los estudiantes.

\section{Currículo como educación personalizada}

Quien más utiliza este término es García Hoz, que en vida fue uno de los pedagogos más importantes en España, e inclusive elaboró un Tratado de educación personalizada, obra dividida en treinta y tres volúmenes, realizada en colaboración con profesores europeos y americanos, que terminó en 1997. Intenta así abordar las características que debe presentar el currículo en una educación personalizada, cada volumen aborda temas como la 
organización de la clase, el papel del docente y del estudiante, brindando información muy importante para el análisis.

Se puede apreciar en el discurso de García Hoz (1988b) respecto a los temas mencionados en su tratado una serie de características de la acción educativa tales como la reflexión, la creatividad y el optimismo entre otras, que hacen de esta propuesta una novedosa forma de ver la práctica en las aulas.

En la educación personalizada García Hoz (1988b) no solo habla de ver al estudiante como persona, sino de recordar que el docente también lo es y que su estilo de enseñanza y la forma en que interpreta la realidad van de una u otra forma a influir en su intervención en el aula. Teniendo en cuenta lo anterior se señala que dicha situación no puede dar paso a que el proceso educativo se convierta en una simple repetición de la cultura, sino que debe manifestarse en el aula la iniciativa propia de la persona humana, convirtiendo la educación personalizada en un estilo propio que se manifiesta en características muy concretas.

En resumen, indica García $\mathrm{Hoz}$ (1988b, p. 30) que son cuatro características las que definen el estilo de la educación personalizada:

\footnotetext{
Es integradora y abierta.

Es reflexiva y creadora.

Es singularizadora y convivencial.

Es optimista.
}

A continuación se explica cada una de las características que se le atribuyen al estilo propio de la educación personalizada.

Respecto a que el estilo de la educación personalizada es integrador y abierto, se considera esta característica como fundamental, que nace como consecuencia de la gran variedad de tareas que exigen al docente una atención dispersa y agotadora.

Indica García Hoz (1988b) que la educación corre el riesgo de convertirse en la suma de gran cantidad de actividades y de aprendizajes sin conexión e incompletos que, en lugar de integrar a la persona, la disgrega, obstaculizando el sentido de la vida y debilitando la capacidad del estudiante de ordenar su vida en medio de esa multitud de exigencias, por lo que la educación personalizada debe contribuir a solventar esta situación.

En cuanto a que el estilo de la educación personalizada es reflexivo y creador se caracteriza de esta manera pues se considera que el acto educativo debe llegar al fondo de la persona, lo que exige en el docente una reflexión sobre sí mismo, trasladando la atención del mundo exterior para fijarla a sus propias operaciones de pensamiento.

Continúa García Hoz (1988b) señalando que la reflexión, en la medida en que es una mirada hacia el interior de la persona para elaborar los datos obtenidos de la observación externa, formular juicios, establecer con creatividad nuevas relaciones, tomar decisiones sobre las limitantes del ambiente, es el componente típico que hace humano a un aprendizaje y le diferencia por lo tanto del aprendizaje animal.

La educación personalizada según García Hoz (1988b) debe tener un carácter singularizador, necesario en el mundo de hoy, pues existe una fuerte presión masificadora por parte de la sociedad en que se vive.

Es necesario desde esta perspectiva ver al estudiante como una persona única que necesita ser atendida de manera especial y a partir de esa atención singular buscar la mejoría del grupo, pero sin olvidar que no se puede pensar primero en el colectivo, pues se corre el riesgo de atropellar lo individual.

Como aspecto importante cita García Hoz (1988b) que el atender de manera individual a cada persona no significa que existe un descuido del desarrollo de las capacidades sociales, es precisamente el reconocer las diferencias de cada individuo lo que debe aprovecharse para enriquecer la totalidad del grupo. Presenta así este 
autor un ejemplo que ilustra muy bien este punto al señalar que una orquesta se enriquece con la ejecución que cada músico hace durante el concierto, pero que tener veinte contrabajos ejecutándose al mismo tiempo, dando la misma nota, no aporta la misma espectacularidad.

Por último en lo que se refiere a las características de estilo se explica que la educación personalizada es optimista y que se debe reflejar en un tono vital del profesor que se manifiesta en su actitud y actividad.

Menciona García Hoz (1988b) que este optimismo se hace presente en el momento mismo en que el docente inicia su relación con los estudiantes, pues desde el contacto inicial la presencia del profesor ejerce una influencia en la actitud de los estudiantes.

Lo anterior se presentará según García Hoz (1988b) a lo largo de la relación docente-estudiante y se manifestará en todos los aspectos de la comunicación, incluso en la no verbal.

El optimismo del que se habla en la educación personalizada, no responde a una actitud de rehuir a la realidad y a los problemas, sino que hace un llamado a fortalecer una visión completa de la realidad, observando en ella todos los aspectos positivos y negativos que existen para sacarles provecho en búsqueda de la mejora personal y colectiva.
Por último un aspecto importante de indicar en la educación personalizada es que al lado de cada una de las características anteriores se encuentra una característica que se presenta de manera transversal.

Dicha característica hace referencia que al hablar de valores se hace desde el humanismo cristiano, tratando de llevar éstos a un plano más amplio y universal, por lo que este elemento se presentará de manera implícita dentro de todo el proceso curricular.

\section{Comparación de las distintas posiciones expuestas}

A continuación se presentan tres cuadros donde se comparan las anteriores posiciones tomando como punto de partida lo que se refiere al papel del estudiante, el papel del docente y el efecto en las características de las situaciones de aprendizaje que se vayan a sugerir y de esta manera tener una visión sistemática del enfoque curricular centrado en la persona, que a su vez sirva de insumo a quienes se interesen en el tema.

En primer lugar se presentan el papel del estudiante desde las cuatro exposiciones.

Al comparar cada una de las posiciones, se puede señalar que el papel del

\begin{tabular}{|c|c|c|c|c|}
\hline & 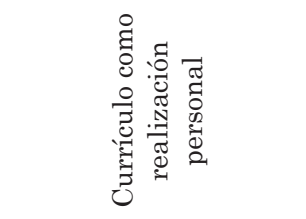 & 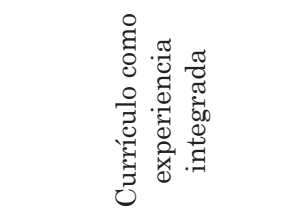 & 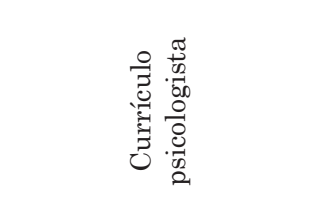 & 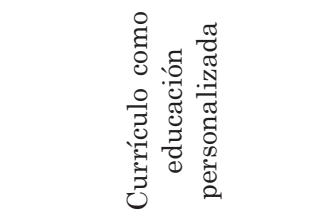 \\
\hline 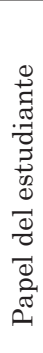 & $\begin{array}{l}\text { Juega un papel activo, } \\
\text { pues debe reflejar sus } \\
\text { aprendizajes en el } \\
\text { desarrollo de su } \\
\text { entorno próximo. }\end{array}$ & $\begin{array}{l}\text { Aunque tiene la guía } \\
\text { del docente el } \\
\text { estudiante debe } \\
\text { participar } \\
\text { aprovechando al } \\
\text { máximo las } \\
\text { experiencias escolares } \\
\text { en su desarrollo } \\
\text { integral. }\end{array}$ & $\begin{array}{l}\text { A la población } \\
\text { estudiantil se le da la } \\
\text { oportunidad de } \\
\text { decidir, que mueva sus } \\
\text { propios recursos y se } \\
\text { responsabilice de lo } \\
\text { que va a aprender. }\end{array}$ & $\begin{array}{l}\text { Centra el proceso } \\
\text { educativo en la persona, } \\
\text { convierte al estudiante } \\
\text { en protagonista de sus } \\
\text { aprendizajes, dentro de } \\
\text { un ambiente optimista } \\
\text { y procurando atender } \\
\text { las distintas demandas } \\
\text { sociales desde una } \\
\text { perspectiva integral. }\end{array}$ \\
\hline
\end{tabular}


estudiante desde un currículo centrado en la persona, se encamina a que este sea activo, el estudiante debe sentirse comprometido con sus aprendizajes, encontrando la utilidad de los mismos de diversas maneras, aplicándolos en su entorno próximo, proponiendo temas de su interés, participando de discusiones o simplemente trabajando de manera independiente en lo que se le solicita, formarse en la idea de que debe buscar soluciones apropiadas, en el marco de una serie de valores universales e ir dependiendo menos de la presencia de un adulto en el cumplimiento de sus deberes.

En segundo lugar se expone el papel del docente, del cual se puede apreciar lo siguiente:

En un currículo centrado en la persona, el docente acompaña al estudiante

\begin{tabular}{|c|c|c|c|c|}
\hline & 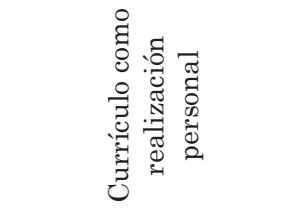 & 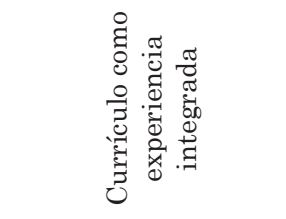 & 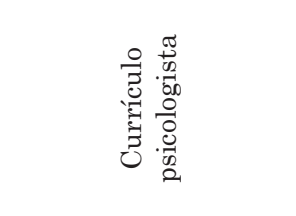 & 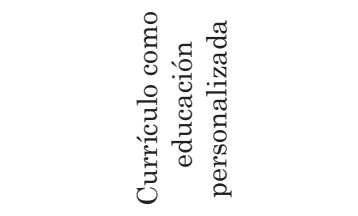 \\
\hline 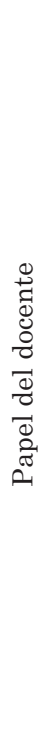 & $\begin{array}{l}\text { Es un guía que } \\
\text { acompaña el proceso } \\
\text { de formación. }\end{array}$ & $\begin{array}{l}\text { Juega un papel de } \\
\text { guía en el proceso de } \\
\text { formación, } \\
\text { propiciador de } \\
\text { experiencias que } \\
\text { abarquen de manera } \\
\text { integral al } \\
\text { estudiante y le } \\
\text { permitan su } \\
\text { aprendizaje. }\end{array}$ & $\begin{array}{l}\text { Se sugiere un } \\
\text { profesor que no } \\
\text { utilice recetas } \\
\text { estereotipadas, pues } \\
\text { la práctica } \\
\text { educativa es } \\
\text { dinámica, se requiere } \\
\text { un docente activo, } \\
\text { que se } \\
\text { mantenga al lado } \\
\text { de los estudiantes, } \\
\text { que actúe de manera } \\
\text { innovadora, que sea } \\
\text { auténtico y no un } \\
\text { simple consumidor } \\
\text { del currículo. }\end{array}$ & $\begin{array}{l}\text { El profesor se convierte } \\
\text { para cada estudiante en la } \\
\text { ayuda, el } \\
\text { consejero, la persona, que } \\
\text { no sólo } \\
\text { permite la adquisición del } \\
\text { saber, o del saber hacer, el } \\
\text { que le enseñe cosas, sino } \\
\text { el que le permite, al hacer } \\
\text { esto, descubrir con } \\
\text { alegría sus propias } \\
\text { aptitudes, sus verdaderas } \\
\text { posibilidades de progreso. } \\
\text { En esta perspectiva se } \\
\text { menciona que el docente } \\
\text { es también persona y que } \\
\text { esto es de suma } \\
\text { importancia en el } \\
\text { desarrollo del enfoque } \\
\text { curricular centrado en la } \\
\text { persona. }\end{array}$ \\
\hline
\end{tabular}

en su aprendizaje, le brinda situaciones con las que pueda ir convirtiéndose en protagonista en el día a día, creciendo en lo cognitivo tanto como en lo físico y en lo emocional.

Se ve al estudiante como una persona "capaz de recibir estímulos, capaz de crear y capaz de descubrir" (García Hoz, 1988a, p. 93).

El docente como persona debe estar consciente de sus puntos fuertes y débiles para que al acompañar a los estudiantes en el proceso de formación se convierta en una orientación apropiada. Debe ser flexible para que en medio de las exigencias que se le solicitan, no pierda de vista su norte en el día a día con la población estudiantil que atiende.

Muy importante es que no se pierda en el colectivo y las estadísticas, para que se preocupe por ayudar a todos los niños y niñas en la mejora personal de cada uno. 
Algunas ideas que se presentan para conseguir lo anterior en la educación personalizada indican la necesidad de que el docente realice un diagnóstico que le permita conocer las aptitudes, los conocimientos, la emotividad, los intereses de cada persona del aula, ya que cada uno de los aspectos anteriores debe ser tomado en cuenta en la programación de las actividades escolares. Pero al lado de estos aspectos se indica la necesidad de conocer y evaluar el contorno social.

Por último se presentan las características que deben estar presentes en las situaciones de aprendizaje que se desarrollen,

\begin{tabular}{|c|c|c|c|c|}
\hline & 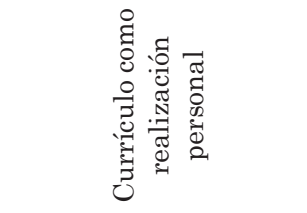 & 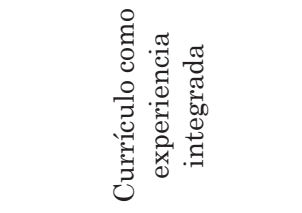 & 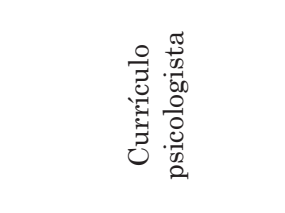 & 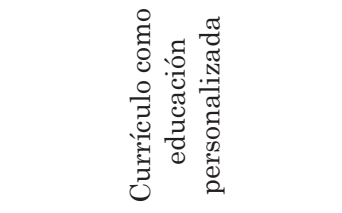 \\
\hline 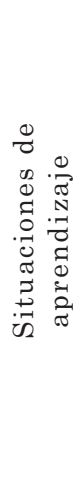 & $\begin{array}{l}\text { Se orientan en } \\
\text { términos de las } \\
\text { necesidades de la } \\
\text { persona para alcanzar } \\
\text { su desarrollo. }\end{array}$ & $\begin{array}{l}\text { Se concentran } \\
\text { en la búsqueda } \\
\text { de experiencias } \\
\text { personales, integrales } \\
\text { que sean de provecho } \\
\text { para el aprendizaje } \\
\text { individual. }\end{array}$ & $\begin{array}{l}\text { Deben permitir } \\
\text { que los estudiantes } \\
\text { consideren los temas } \\
\text { de la clase como algo } \\
\text { importante para sus } \\
\text { objetivos personales. }\end{array}$ & $\begin{array}{l}\text { Deben buscar humanizar } \\
\text { a hombres, mujeres, } \\
\text { niños y niñas. Deben } \\
\text { partir de creer en el } \\
\text { estudiante como una } \\
\text { persona original, } \\
\text { creativa, única e } \\
\text { irrepetible. La finalidad } \\
\text { de la metodología } \\
\text { personalizante es servir } \\
\text { de guía y orientación } \\
\text { al desarrollo de la } \\
\text { personalidad de los } \\
\text { estudiantes. }\end{array}$ \\
\hline
\end{tabular}

Las situaciones de aprendizaje en el enfoque curricular centrado en la persona no es un fin en sí misma, sino un medio por el cual el docente y el estudiante tienen la oportunidad de crecer como personas.

No existe una receta de cómo debe ser la metodología dentro de este enfoque, pues sea cual fuese, lo que interesa es que corresponda a la adecuada para el grupo y al mismo tiempo para cada persona, permitiéndole crecer de una manera original, única e irrepetible.

Las actividades de aprendizaje podrían desarrollarse en medio de las características que señala García Hoz (1988b) la integración y la apertura, la reflexión y la creación, lo singular sin olvidar la convivencia y el optimismo en el desarrollo de las clases.
En la educación centrada en la persona se indica que la agrupación de los alumnos será un condicionante del aprendizaje, pues a partir de esta se establecerán una serie distinta de relaciones personales a través de la comunicación, estas relaciones afectarán tanto a la persona que aprende como a los medios que se han de utilizar.

Se indica por parte de García $\mathrm{Hoz}$ (1988a) que el éxito en la atención de los estudiantes no dependerá de la cantidad de estudiantes sino en la posibilidad de que cada alumno pueda, en ocasiones, trabajar individualmente, en otras, en colaboración con grupos pequeños o en grupos de mayor amplitud.

En un enfoque centrado en la persona no se habla así de una agrupación de 
alumnos rígida que responda a todas las necesidades de las personas en la clase.

En resumen se puede señalar que la educación personalizada en cuanto a lo metodológico aborda cuatro aspectos muy importantes: la adecuación a la singularidad de la personalidad de cada estudiante armonizándola con las formas cooperativas de trabajo, la unificación del trabajo escolar en la actividad expresiva, la flexibilidad en la programación y utilización de las situaciones de aprendizaje y por último a la posibilidad de elección de contenido y técnicas de trabajo por parte del alumno.

\section{El enfoque curricular centrado en la persona, una nueva alternativa}

Como se señaló anteriormente, todo enfoque curricular va a orientar la práctica docente, dicha orientación se ve reflejada en diversos momentos, en el presente artículo se han señalado tres, que inciden directamente en el quehacer educativo, éstos son la metodología, el papel del docente y el papel del estudiante.

La intención ha sido contribuir con las características de un enfoque curricular, que de orientar la práctica, permita a los involucrados tener claro cómo debe ser su participación dentro del proceso educativo, de una manera coherente inclusive con lo propuesto por el Ministerio de Educación Pública, pues las características de este enfoque están acordes con lo señalado en los fines de la educación costarricense.

El docente al iniciar sus labores cuenta con los planes de estudio que proponen un determinado enfoque curricular, sin embargo, no tiene un respaldo teórico que le permita comprender y trasladar lo que sabe a aspectos prácticos respecto al accionar en su realidad, cuestión que se busca solventar con el presente documento, para aquellos que elijan este enfoque curricular como su eje.
Se retoma además la idea de ver la metodología como un medio para crecer como persona, lo cual no se expresa en la propuesta oficial del MEP, otro elemento importante del presente trabajo, pues el docente antes de pensar en los caminos que él desea trazar a sus estudiantes, debe pensar en si son realmente los que ellos y ellas necesitan para ser mejores personas y por lo tanto mejores ciudadanos.

Para futuros trabajos de curriculistas y docentes se cuenta con un documento único, en el sentido de que no se ha sistematizado antes los aspectos relacionados al enfoque curricular centrado en la persona, con lo cual pueden encontrarse una serie de elementos orientadores en cuanto a términos y propuestas, lo que será de gran beneficio en cuanto a la búsqueda de información y a la vez se contribuye al disminuir confusiones en cuanto al enfoque se refiere.

El enfoque curricular centrado en la persona es un enfoque que al plantear la metodología como un medio para crecer de manera integral, no presenta inconvenientes para ser aplicado en cualquier área del aprendizaje y que responde de manera muy pertinente a la concepción de educación costarricense, donde más allá de lo cognitivo, interesa formar personas amantes de su patria e interesadas por crecer en medio del servicio a los demás. Para futuros trabajos se puede indagar qué instituciones utilizan este enfoque curricular y contrastar por medio de un estudio cuantitativo con otras instituciones que emplean otros enfoques curriculares y obtener conclusiones al respecto.

Lo importante es que este es un paso para aquellas personas que quieran hacer un cambio significativo en su entorno inmediato, teniendo en cuenta que la claridad y dominio de lo que se hace impactará de manera positiva la práctica educativa.

En educación no existen recetas de laboratorio, el enfoque curricular centrado en la persona es una opción entre otras que a diferencia de enfoques tecnológicos centrados en la consecución de objetivos de producción como lo hace una empresa, se 
preocupa por desarrollar en la educación formal un verdadero proceso que busca el desarrollo y perfección no solo de conocimientos y aptitudes, sino del fortalecimiento y desenvolvimiento pleno de la persona en sus múltiples manifestaciones.

Se pretende así que todo lo que se haga en las instituciones educativas contribuya a que las personas presentes en ellas sean conscientes de sus actos y que en ellos afirme su libertad personal y asuma la responsabilidad de sus acciones, siendo esto un gran aporte para la sociedad.

\section{Referencias bibliográficas}

Buitrago, O. y Amaya, B. L. (2001). Educación personalizada una modalidad educativa. Revista de Ciencias Humanas, 26. Extraído el 25 de marzo de 2007 de http://www.utp. edu.co/ chumanas/revistas/revistas/ rev26/buitrago.htm

Castillo, L. (2003). Enfoques ó concepciones curriculares. Extraído el miércoles 4 de abril de 2007 de

http://www.asesoriaspedagogicas.cl/ index_ipp.htm

Chadwick, C. (1987). Currículum. Enfoques curriculares. En C. Carvajal (Comp.), Curriculum como realización personal (pp. 1-6). San José, Costa Rica, Facultad de Educación. Escuela de Formación Docente. Universidad de Costa Rica.

Coll, C. (1991). Psicología y curriculum. Una aproximación psicopedagógica a la elaboración del currículum escolar. Barcelona: Ediciones Paidós Ibérica, S.A.

Díaz, A. (1997). Didáctica y currículum. Convergencias en los programas de estudio. México, D.F.: Editoral Paidós Mexicana, S.A.

García, J. (s.f.). Paradigma humanista en la educación y Carl Rogers. Extraído el 30 de abril de 2007 de http://www.psicopedagogia.com/ articulos $/$ articulo $=355$

García, V. (1988a). Educación personalizada. Madrid: Ediciones Rialp.

García, V. (1988b). La práctica de la educación personalizada. Madrid: Ediciones Rialp.

García, V. (1993). Introducción General a una pedagogía de la persona. Madrid: Ediciones Rialp.

Ministerio de Educación Pública [MEP]. (s.f.). Ley Fundamental de Educación $N^{o}$ 2160. Capítulo I: De los Fines. Artículo $2^{\circ}$. Extraído el 4 abril de 2007 de http://www.mep.go.cr/ CentroDeInformacion/Reglamentos. $\underline{\operatorname{aspx}}$

Molina, Z. (2004). Planeamiento didáctico: fundamentos, principios, estrategias y procedimientos para el desarrollo. (7 ${ }^{\circ}$ Reimpresión). San José, Costa Rica: EUNED.

Palladino, E. (1998). Diseños curriculares y calidad educativa. Buenos Aires: Espacio Editorial.

Villarini, A. (1996). El currículo orientado al desarrollo humano integral. Río Piedra, Puerto Rico: Biblioteca del Pensamiento Crítico.

Zabalza, M. (1997). Diseño y desarrollo curricular $\left(7^{\circ}\right.$ ed.). Madrid: Ediciones Narcea, S.A. 